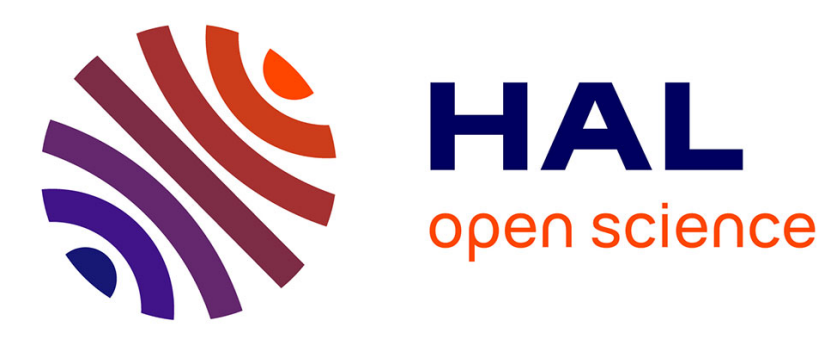

\title{
Multipliers of uniform topological algebras
}

Mohammed El Azhari

\section{To cite this version:}

Mohammed El Azhari. Multipliers of uniform topological algebras. Annales Mathematicae Silesianae, 2017, 31, pp.71-81. 10.1515/amsil-2017-0002 . hal-01355345v2

\section{HAL Id: hal-01355345 \\ https://hal.science/hal-01355345v2}

Submitted on 22 Jan 2017

HAL is a multi-disciplinary open access archive for the deposit and dissemination of scientific research documents, whether they are published or not. The documents may come from teaching and research institutions in France or abroad, or from public or private research centers.
L'archive ouverte pluridisciplinaire HAL, est destinée au dépôt et à la diffusion de documents scientifiques de niveau recherche, publiés ou non, émanant des établissements d'enseignement et de recherche français ou étrangers, des laboratoires publics ou privés. 


\title{
Multipliers of uniform topological algebras
}

\author{
M. El Azhari
}

\begin{abstract}
Let $E$ be a complete uniform topological algebra with Arens-Michael normed factors $\left(E_{\alpha}\right)_{\alpha \in \Lambda}$. Then $M(E) \cong \lim M\left(E_{\alpha}\right)$ within an algebra isomorphism $\varphi$. If each factor $E_{\alpha}$ is complete, then every multiplier of $E$ is continuous and $\varphi$ is a topological algebra isomorphism where $M(E)$ is endowed with its seminorm topology.
\end{abstract}

Mathematics Subject Classification 2010: 46H05, 46K05.

Keywords: Multiplier algebra, locally $m$-pseudoconvex algebra, uniform topological algebra.

\section{Preliminaries}

A topological algebra is an algebra (over the complex field) which is also a Hausdorff topological vector space such that the multiplication is separately continuous. For a topological algebra $E$, we denote by $\Delta(E)$ the set of all nonzero continuous multiplicative linear functionals on $E$. An approximate identity in a topological algebra $E$ is a net $\left(e_{\omega}\right)_{\omega \in \Omega}$ such that for each $x \in E$ we have $x e_{\omega} \rightarrow_{\omega} x$ and $e_{\omega} x \rightarrow_{\omega} x$. Let $E$ be an algebra, a function $p: E \rightarrow[0, \infty[$ is called a pseudo-seminorm, if there exists $0 \lessgtr k \leq 1$ such that $p(x+y) \leq$ $p(x)+p(y), p(\lambda x)=|\lambda|^{k} p(x)$ and $p(x y) \leq \bar{p}(x) p(y)$ for all $x, y \in E$ and $\lambda \in \mathbb{C}$. $k$ is called the homogenity index of $p$. If $k=1, p$ is called a seminorm. A pseudo-seminorm $p$ is a pseudo-norm, if $p(x)=0$ implies $x=0$. A locally $m$-pseudoconvex algebra is a topological algebra $E$ whose topology is determined by a directed family $\left\{p_{\alpha}: \alpha \in \Lambda\right\}$ of pseudo-seminorms. For each $\alpha \in$ $\Lambda, \operatorname{ker}\left(p_{\alpha}\right)=\left\{x \in E: p_{\alpha}(x)=0\right\}$, the quotient algebra $E_{\alpha}=E / \operatorname{ker}\left(p_{\alpha}\right)$ is a pseudo-normed algebra in the pseudo-norm $\bar{p}_{\alpha}\left(x_{\alpha}\right)=p_{\alpha}(x), x_{\alpha}=x+\operatorname{ker}\left(p_{\alpha}\right)$. Let $f_{\alpha}: E \rightarrow E_{\alpha}, f_{\alpha}(x)=x+\operatorname{ker}\left(p_{\alpha}\right)=x_{\alpha}$, be the quotient map, $f_{\alpha}$ is a continuous homomorphism from $E$ onto $E_{\alpha}$. We endow the set $\Lambda$ with the partial order: $\alpha \leq \beta$, if and only, if $p_{\alpha}(x) \leq p_{\beta}(x)$ for all $x \in E$. Take $\alpha \leq \beta$ in $\Lambda$, since $\operatorname{ker}\left(p_{\beta}\right) \subset \operatorname{ker}\left(p_{\alpha}\right)$, we define the surjective continuous homomorphism $f_{\alpha \beta}: E_{\beta} \rightarrow E_{\alpha}, x_{\beta}=x+\operatorname{ker}\left(p_{\beta}\right) \rightarrow x_{\alpha}=x+\operatorname{ker}\left(p_{\alpha}\right)$. Thus $\left\{\left(E_{\alpha}, f_{\alpha \beta}\right), \alpha \leq \beta\right\}$ is a projective system of pseudo-normed algebras. We also define the algebra isomorphism (into) $\Phi: E \rightarrow \lim E_{\alpha}, \Phi(x)=\left(f_{\alpha}(x)\right)_{\alpha \in \Lambda}$, the canonical projections $\pi_{\alpha}: \prod_{\alpha \in \Lambda} E_{\alpha} \rightarrow E_{\alpha}$ and the restrictions to the projective limit $g_{\alpha}=\pi_{\alpha} / \lim _{\leftarrow} E_{\alpha}: \lim E_{\alpha} \rightarrow E_{\alpha}$. Since $g_{\alpha} \circ \Phi=f_{\alpha}$ and the quotient map $f_{\alpha}$ is surjective, it follows that the map $g_{\alpha}$ is surjective, this proves that the projective system $\left\{\left(E_{\alpha}, f_{\alpha \beta}\right), \alpha \leq \beta\right\}$ is perfect in the sense of [5, Definition 2.10] (see also [2, Definition 2.7]). Thus, if $E$ is a locally $m$-pseudoconvex algebra (not necessarly complete), then its generalized Arens-Michael projective 
system $\left\{\left(E_{\alpha}, f_{\alpha \beta}\right), \alpha \leq \beta\right\}$ is perfect. If $E$ is complete, then $E \cong \varliminf E_{\alpha}$ within a topological algebra isomorphism.

A locally $m$-convex algebra is a topological algebra $E$ whose topology is defined by a directed family $\left\{p_{\alpha}: \alpha \in \Lambda\right\}$ of seminorms. For each $\alpha \in \Lambda$, put $\Delta_{\alpha}(E)=$ $\left\{f \in \Delta(E):|f(x)| \leq p_{\alpha}(x), x \in E\right\}$. Let $E$ be an algebra with involution $*$. A seminorm on $E$ is called a $C^{*}$-seminorm if $p\left(x^{*} x\right)=p(x)^{2}$ for all $x \in E$. A complete locally $m$-convex *-algebra $\left(E,\left(p_{\alpha}\right)_{\alpha \in \Lambda}\right)$, for which each $p_{\alpha}$ is a $C^{*}$ seminorm, is called a locally $C^{*}$-algebra. A uniform seminorm on an algebra $E$ is a seminorm $p$ satisfying $p\left(x^{2}\right)=p(x)^{2}$ for all $x \in E$. A uniform topological algebra is a topological algebra whose topology is determined by a directed family of uniform seminorms. In that case, such a topological algebra is also named a uniform locally convex algebra. A uniform normed algebra is a normed algebra $(E,\|\|$.$) such that \left\|x^{2}\right\|=\|x\|^{2}$ for all $x \in E$.

An algebra $E$ is called proper if for any $x \in E, x E=E x=\{0\}$ implies $x=0$. If $E$ has identity, then $E$ is proper. Moreover, a topological algebra with approximate identity is proper. Also, a (Hausdorff) uniform topological algebra is proper. Let $E$ be an algebra, a map $T: E \rightarrow E$ is called a multiplier if $T(x) y=x T(y)$ for all $x, y \in E$. We denote by $M(E)$ the set of all multipliers of $E$. It is known that if $E$ is a proper algebra, then any multiplier $T$ of $E$ is linear with the property $T(x y)=T(x) y=x T(y)$ for all $x, y \in E$, and $M(E)$ is a commutative algebra with the identity map $I$ of $E$ as its identity. Let $(E,\|\|$. be a uniform normed algebra, and let $M_{c}(E)$ be the algebra of all continuous multipliers of $E$ with the operator norm $\|\cdot\|_{o p}$. It is known that $\|\cdot\|_{o p}$ has the square property and the map $l:(E,\|\cdot\|) \rightarrow\left(M_{c}(E),\|\cdot\|_{o p}\right), l(x)(y)=x y$, is an isometric isomorphism (into). For information on the multiplier algebra in non-normed topological algebras, see also [3] and [4].

In the sequel, we will need the following elementary result called the universal property of the quotient: Let $X, Y, Z$ be vector spaces, $f: X \rightarrow Y$ and $g: X \rightarrow$ $Z$ be linear maps. If the map $g$ is surjective and $\operatorname{ker}(g) \subset \operatorname{ker}(f)$, then there exists a unique linear map $h: Z \rightarrow Y$ such that $f=h \circ g$.

\section{Results}

Proposition 2.1. Let $\left(E,\left(p_{\alpha}\right)_{\alpha \in \Lambda}\right)$ be a locally m-pseudoconvex algebra with proper pseudo-normed factors $\left(E_{\alpha}\right)_{\alpha \in \Lambda}$. The following assertions are equivalent: (i) $T\left(k e r\left(f_{\alpha}\right)\right) \subset \operatorname{ker}\left(f_{\alpha}\right)$ for all $T \in M(E)$ and $\alpha \in \Lambda$;

(ii) for each $T \in M(E)$, there exists a unique $\left(T_{\alpha}\right)_{\alpha \in \Lambda} \in \prod_{\alpha \in \Lambda} M\left(E_{\alpha}\right)$ such that $f_{\alpha} \circ T=T_{\alpha} \circ f_{\alpha}$ and $T_{\alpha} \circ f_{\alpha \beta}=f_{\alpha \beta} \circ T_{\beta}$ for all $\alpha \leq \beta$ in $\Lambda$. Furthermore, $T$ is continuous if and only if $T_{\alpha}$ is continuous for all $\alpha \in \Lambda$.

Proof. Since the pseudo-normed factors $\left(E_{\alpha}\right)_{\alpha \in \Lambda}$ are proper, it follows that the algebra $E$ is proper and so every multiplier of $E$ (or $E_{\alpha}$ ) is linear.

(ii) $\Rightarrow(i)$ : If $T \in M(E)$ and $x \in \operatorname{ker}\left(f_{\alpha}\right)$, then $f_{\alpha}(T(x))=T_{\alpha}\left(f_{\alpha}(x)\right)=0$ and so $T(x) \in \operatorname{ker}\left(f_{\alpha}\right)$.

$(i) \Rightarrow($ ii $)$ : Take $T \in M(E)$ and $\alpha \in \Lambda$. Since $T\left(k e r\left(f_{\alpha}\right)\right) \subset \operatorname{ker}\left(f_{\alpha}\right)$ and 
by using the universal property of the quotient (see Preliminaries), there exists a unique linear map $T_{\alpha}: E_{\alpha} \rightarrow E_{\alpha}$ such that $f_{\alpha} \circ T=T_{\alpha} \circ f_{\alpha}$. Let $\alpha \in \Lambda$ and $x, y \in E, T_{\alpha}\left(f_{\alpha}(x) f_{\alpha}(y)\right)=T_{\alpha}\left(f_{\alpha}(x y)\right)=f_{\alpha}(T(x y))=f_{\alpha}(x T(y))=$ $f_{\alpha}(x) f_{\alpha}(T(y))=f_{\alpha}(x) T_{\alpha}\left(f_{\alpha}(y)\right)$ and similary on the other side, so $T_{\alpha}$ is a multiplier of $E_{\alpha}$. Let $\alpha \leq \beta$ in $\Lambda$, we have $T_{\alpha} \circ f_{\alpha}=f_{\alpha} \circ T$, then $T_{\alpha} \circ f_{\alpha \beta} \circ f_{\beta}=$ $f_{\alpha \beta} \circ f_{\beta} \circ T=f_{\alpha \beta} \circ T_{\beta} \circ f_{\beta}$, hence $T_{\alpha} \circ f_{\alpha \beta}=f_{\alpha \beta} \circ T_{\beta}$ since the quotient map $f_{\beta}$ is surjective. Suppose that $T$ is continuous. Let $O_{\alpha}$ be an open set in $E_{\alpha}$, we have $f_{\alpha}^{-1}\left(T_{\alpha}^{-1}\left(O_{\alpha}\right)\right)=\left(T_{\alpha} \circ f_{\alpha}\right)^{-1}\left(O_{\alpha}\right)=\left(f_{\alpha} \circ T\right)^{-1}\left(O_{\alpha}\right)$ which is open in $E$ since $f_{\alpha} \circ T$ is continuous, then $T_{\alpha}^{-1}\left(O_{\alpha}\right)$ is open in $E_{\alpha}$. Conversely, suppose that $T_{\alpha}$ is continuous for all $\alpha \in \Lambda$. Since $E$ is topologically isomorphic to a subalgebra of $\lim E_{\alpha}, T$ is continuous if and only if $f_{\alpha} \circ T$ is continuous for all $\alpha \in \Lambda$. Since $\overleftarrow{f_{\alpha}} \circ T=T_{\alpha} \circ f_{\alpha}$ and $T_{\alpha}$ is continuous for all $\alpha \in \Lambda$, we deduce that $T$ is continuous.

Proposition 2.2. Let $\left(E,\left(p_{\alpha}\right)_{\alpha \in \Lambda}\right)$ be a locally m-pseudoconvex algebra with proper pseudo-normed factors $\left(E_{\alpha}\right)_{\alpha \in \Lambda}$. The following assertions are equivalent: (j) $U\left(\operatorname{ker}\left(f_{\alpha \beta}\right)\right) \subset \operatorname{ker}\left(f_{\alpha \beta}\right)$ for all $U \in M\left(E_{\beta}\right)$ and $\alpha \leq \beta$ in $\Lambda$;

(jj) there exists a unique projective system $\left\{\left(M\left(E_{\alpha}\right), h_{\alpha \beta}\right), \alpha \leq \beta\right\}$ such that $h_{\alpha \beta}(U) \circ f_{\alpha \beta}=f_{\alpha \beta} \circ U$ for all $U \in M\left(E_{\beta}\right)$ and $\alpha \leq \beta$ in $\Lambda$. Furthermore, if $E_{\alpha}$ is complete for all $\alpha \in \Lambda$, then $h_{\alpha \beta}$ is continuous for all $\alpha \leq \beta$ in $\Lambda$.

Proof. $(j j) \Rightarrow(j)$ : Let $U \in M\left(E_{\beta}\right)$ and $x_{\beta} \in \operatorname{ker}\left(f_{\alpha \beta}\right)$, then $f_{\alpha \beta}\left(U\left(x_{\beta}\right)\right)=$ $h_{\alpha \beta}(U)\left(f_{\alpha \beta}\left(x_{\beta}\right)\right)=0$ and so $U\left(x_{\beta}\right) \in \operatorname{ker}\left(f_{\alpha \beta}\right)$.

$(j) \Rightarrow(j j)$ : Let $\alpha \leq \beta$ in $\Lambda$ and $U \in M\left(E_{\beta}\right)$. Since $U\left(k e r\left(f_{\alpha \beta}\right)\right) \subset \operatorname{ker}\left(f_{\alpha \beta}\right)$ and by using the universal property of the quotient (see Preliminaries), there exists a unique linear map $V: E_{\alpha} \rightarrow E_{\alpha}$ such that $V \circ f_{\alpha \beta}=f_{\alpha \beta} \circ U$. Let $x_{\alpha}=x+\operatorname{ker}\left(p_{\alpha}\right), y_{\alpha}=y+\operatorname{ker}\left(p_{\alpha}\right) \in E_{\alpha}$ where $x, y \in E$. Put $x_{\beta}=x+\operatorname{ker}\left(p_{\beta}\right)$ and $y_{\beta}=y+\operatorname{ker}\left(p_{\beta}\right)$, clearly $x_{\beta}, y_{\beta} \in E_{\beta}$. By definition of the map $f_{\alpha \beta}$, we get $f_{\alpha \beta}\left(x_{\beta}\right)=x_{\alpha}$ and $f_{\alpha \beta}\left(y_{\beta}\right)=y_{\alpha}$. We have $V\left(x_{\alpha} y_{\alpha}\right)=V\left(f_{\alpha \beta}\left(x_{\beta}\right) f_{\alpha \beta}\left(y_{\beta}\right)\right)=$ $V\left(f_{\alpha \beta}\left(x_{\beta} y_{\beta}\right)\right)=f_{\alpha \beta}\left(U\left(x_{\beta} y_{\beta}\right)\right)=f_{\alpha \beta}\left(x_{\beta} U\left(y_{\beta}\right)\right)=f_{\alpha \beta}\left(x_{\beta}\right) f_{\alpha \beta}\left(U\left(y_{\beta}\right)\right)=$ $f_{\alpha \beta}\left(x_{\beta}\right) V\left(f_{\alpha \beta}\left(y_{\beta}\right)\right)=x_{\alpha} V\left(y_{\alpha}\right)$ and similary on the other side, so $V$ is a multiplier of $E_{\alpha}$. This shows the existence of the map $h_{\alpha \beta}: M\left(E_{\beta}\right) \rightarrow M\left(E_{\alpha}\right)$ such that $h_{\alpha \beta}(U) \circ f_{\alpha \beta}=f_{\alpha \beta} \circ U$ for all $U \in M\left(E_{\beta}\right)$ and $\alpha \leq \beta$ in $\Lambda$. Let $\alpha \leq \beta$ in $\Lambda, U_{1}, U_{2} \in M\left(E_{\beta}\right)$ and $\lambda \in \mathbb{C}, h_{\alpha \beta}\left(U_{1}+\lambda U_{2}\right) \circ f_{\alpha \beta}=f_{\alpha \beta} \circ$ $\left(U_{1}+\lambda U_{2}\right)=\left(f_{\alpha \beta} \circ U_{1}\right)+\lambda\left(f_{\alpha \beta} \circ U_{2}\right)=h_{\alpha \beta}\left(U_{1}\right) \circ f_{\alpha \beta}+\lambda h_{\alpha \beta}\left(U_{2}\right) \circ f_{\alpha \beta}=$ $\left(h_{\alpha \beta}\left(U_{1}\right)+\lambda h_{\alpha \beta}\left(U_{2}\right)\right) \circ f_{\alpha \beta}$, hence

$h_{\alpha \beta}\left(U_{1}+\lambda U_{2}\right)=h_{\alpha \beta}\left(U_{1}\right)+\lambda h_{\alpha \beta}\left(U_{2}\right)$ since $f_{\alpha \beta}$ is surjective. Also,

$h_{\alpha \beta}\left(U_{1} \circ U_{2}\right) \circ f_{\alpha \beta}=f_{\alpha \beta} \circ U_{1} \circ U_{2}=h_{\alpha \beta}\left(U_{1}\right) \circ f_{\alpha \beta} \circ U_{2}=h_{\alpha \beta}\left(U_{1}\right) \circ h_{\alpha \beta}\left(U_{2}\right) \circ f_{\alpha \beta}$, then $h_{\alpha \beta}\left(U_{1} \circ U_{2}\right)=h_{\alpha \beta}\left(U_{1}\right) \circ h_{\alpha \beta}\left(U_{2}\right)$ since $f_{\alpha \beta}$ is surjective. Let $\alpha \leq \beta \leq \gamma$ in $\Lambda$ and $W \in M\left(E_{\gamma}\right),\left(h_{\alpha \beta} \circ h_{\beta \gamma}\right)(W) \circ f_{\alpha \gamma}=h_{\alpha \beta}\left(h_{\beta \gamma}(W)\right) \circ f_{\alpha \beta} \circ f_{\beta \gamma}=$ $f_{\alpha \beta} \circ h_{\beta \gamma}(W) \circ f_{\beta \gamma}=f_{\alpha \beta} \circ f_{\beta \gamma} \circ W=f_{\alpha \gamma} \circ W=h_{\alpha \gamma}(W) \circ f_{\alpha \gamma}$, consequently $\left(h_{\alpha \beta} \circ h_{\beta \gamma}\right)(W)=h_{\alpha \gamma}(W)$ since $f_{\alpha \gamma}$ is surjective. Thus $h_{\alpha \beta} \circ h_{\beta \gamma}=h_{\alpha \gamma}$. Let $\alpha \in \Lambda$, if $E_{\alpha}$ is complete, then every multiplier of $E_{\alpha}$ is continuous. Now by assuming that $E_{\alpha}$ is complete for all $\alpha \in \Lambda$, we will show that $h_{\alpha \beta}$ is continuous for all $\alpha \leq \beta$ in $\Lambda$ (see also, the proof of Theorem 2.12 in [5]). For $\alpha \in \Lambda$ and $r \geqslant 0$, let $B_{\alpha}(0, r)=\left\{x_{\alpha} \in E_{\alpha}: \bar{p}_{\alpha}\left(x_{\alpha}\right) \leq r\right\}$. We denote by $\|\cdot\|_{\alpha}$ the 
operator pseudo-norm on $M\left(E_{\alpha}\right)$. Let $\alpha \leq \beta$ in $\Lambda, f_{\alpha \beta}$ is open by the open mapping theorem, so there is $\lambda \geqslant 0$ such that $\lambda B_{\alpha}(0,1) \subset f_{\alpha \beta}\left(B_{\beta}(0,1)\right)$ i.e. $B_{\alpha}(0,1) \subset f_{\alpha \beta}\left(B_{\beta}(0, r)\right)$ where $r=\lambda^{-k_{\beta}}$ and $k_{\beta}$ is the homogenity index of $\bar{p}_{\beta}$. Let $U \in M\left(E_{\beta}\right)$,

$\left\|h_{\alpha \beta}(U)\right\|_{\alpha}=\sup \left\{\bar{p}_{\alpha}\left(h_{\alpha \beta}(U)\left(f_{\alpha}(x)\right)\right): f_{\alpha}(x) \in B_{\alpha}(0,1)\right\}$

$\leq \sup \left\{\bar{p}_{\alpha}\left(h_{\alpha \beta}(U)\left(f_{\alpha \beta}\left(f_{\beta}(x)\right)\right)\right): f_{\beta}(x) \in B_{\beta}(0, r)\right\}$

$=\sup \left\{\bar{p}_{\alpha}\left(f_{\alpha \beta}\left(U\left(f_{\beta}(x)\right)\right)\right): f_{\beta}(x) \in B_{\beta}(0, r)\right\}$

$\leq \sup \left\{\bar{p}_{\beta}\left(U\left(f_{\beta}(x)\right)\right): f_{\beta}(x) \in B_{\beta}(0, r)\right\}$

$\leq \sup \left\{\|U\|_{\beta} \bar{p}_{\beta}\left(f_{\beta}(x)\right): f_{\beta}(x) \in B_{\beta}(0, r)\right\}$

$=r\|U\|_{\beta}$.

Therefore $h_{\alpha \beta}$ is continuous.

Theorem 2.3. Let $\left(E,\left(p_{\alpha}\right)_{\alpha \in \Lambda}\right)$ be a complete locally m-pseudoconvex algebra with proper pseudo-normed factors $\left(E_{\alpha}\right)_{\alpha \in \Lambda}$. Assume that $E$ satisfies conditions (i) and $(j)$. Then $M(E) \cong \lim M\left(E_{\alpha}\right)$ within an algebra isomorphism $\varphi$. Furthermore, if each factor $E_{\alpha}$ is complete, then every multiplier of $E$ is continuous and $\varphi$ is a topological algebra isomorphism where $M(E)$ is endowed with its pseudo-seminorm topology.

Proof. Take $T \in M(E)$. By Propositions 2.1 and $2.2,\left(T_{\alpha}\right)_{\alpha \in \Lambda} \in \prod_{\alpha \in \Lambda} M\left(E_{\alpha}\right)$, $T_{\alpha} \circ f_{\alpha \beta}=f_{\alpha \beta} \circ T_{\beta}$ and $h_{\alpha \beta}\left(T_{\beta}\right) \circ f_{\alpha \beta}=f_{\alpha \beta} \circ T_{\beta}$ for all $\alpha \leq \beta$ in $\Lambda$. Hence $h_{\alpha \beta}\left(T_{\beta}\right) \circ f_{\alpha \beta}=T_{\alpha} \circ f_{\alpha \beta}$ and consequently $h_{\alpha \beta}\left(T_{\beta}\right)=T_{\alpha}$ since the map $f_{\alpha \beta}$ is surjective. This shows that $\left(T_{\alpha}\right)_{\alpha \in \Lambda} \in \lim M\left(E_{\alpha}\right)$. Thus the map $\varphi: M(E) \rightarrow$ $\lim M\left(E_{\alpha}\right), T \rightarrow\left(T_{\alpha}\right)_{\alpha \in \Lambda}$, is well defined. We will show that $\varphi$ is an algebra isomorphism. Let $T, S \in M(E)$ and $\lambda \in \mathbb{C}, T_{\alpha} \circ f_{\alpha}=f_{\alpha} \circ T$ and $S_{\alpha} \circ f_{\alpha}=f_{\alpha} \circ S$, then $\left(T_{\alpha}+\lambda S_{\alpha}\right) \circ f_{\alpha}=f_{\alpha} \circ(T+\lambda S)$, so $(T+\lambda S)_{\alpha}=T_{\alpha}+\lambda S_{\alpha}$ by Proposition 2.1. Also, $T_{\alpha} \circ S_{\alpha} \circ f_{\alpha}=T_{\alpha} \circ f_{\alpha} \circ S=f_{\alpha} \circ T \circ S$, hence $(T \circ S)_{\alpha}=T_{\alpha} \circ S_{\alpha}$ by Proposition 2.1. Let $T \in M(E)$, if $T_{\alpha}=0$ for all $\alpha \in \Lambda$, then $f_{\alpha} \circ T=T_{\alpha} \circ f_{\alpha}=0$ for all $\alpha \in \Lambda$ and consequently $T=0$. Let $\left(U_{\alpha}\right)_{\alpha \in \Lambda} \in \lim M\left(E_{\alpha}\right)$ and define the map $T=\Phi^{-1} \circ \varliminf_{\lim _{\alpha}} \circ \Phi: E \rightarrow E$ where $\lim _{U_{\alpha}}$ is the multiplier of $\varliminf^{\lim } E_{\alpha}$ defined by $\left(\lim _{\longleftarrow} U_{\alpha}\right)\left(x_{\alpha}\right)_{\alpha}=\left(U_{\alpha}\left(x_{\alpha}\right)\right)_{\alpha}$ and $\Phi: E \rightarrow \underset{\lim }{\longleftarrow} E_{\alpha}$ is the topological algebra isomorphism given by $\Phi(x)=\left(f_{\alpha}(x)\right)_{\alpha}$. Clearly $T$ is a multiplier of $E$, also $f_{\alpha} \circ T=f_{\alpha} \circ \Phi^{-1} \circ \lim _{\longleftarrow} U_{\alpha} \circ \Phi=U_{\alpha} \circ f_{\alpha}$ for all $\alpha \in \Lambda$, so $\varphi(T)=\left(U_{\alpha}\right)_{\alpha}$. If $E_{\alpha}$ is complete for all $\alpha \in \Lambda$, then every multiplier of $E_{\alpha}$ is continuous, hence every multiplier of $E$ is continuous by Proposition 2.1. The pseudo-seminorm topology on $M(E)$ is the topology defined by the family of pseudo-seminorms $q_{\alpha}(T)=\left\|T_{\alpha}\right\|_{\alpha}, \alpha \in \Lambda$, so $\varphi$ is a topological algebra isomorphism.

Proposition 2.4. Let $\left(E,\left(p_{\alpha}\right)_{\alpha \in \Lambda}\right)$ be a locally m-pseudoconvex algebra with approximate identity $\left(e_{\omega}\right)_{\omega \in \Omega}$. Then $E$ satisfies conditions (i) and (j).

Proof. Let $T \in M(E), x \in \operatorname{ker}\left(f_{\alpha}\right)$ and $\omega \in \Omega$,

$f_{\alpha}(T(x))=f_{\alpha}\left(T\left(x-x e_{\omega}+x e_{\omega}\right)\right)$

$=f_{\alpha}\left(T(x)-T\left(x e_{\omega}\right)\right)+f_{\alpha}\left(T\left(x e_{\omega}\right)\right)=f_{\alpha}\left(T(x)-T(x) e_{\omega}\right)+f_{\alpha}\left(x T\left(e_{\omega}\right)\right)$

$=f_{\alpha}\left(T(x)-T(x) e_{\omega}\right)+f_{\alpha}(x) f_{\alpha}\left(T\left(e_{\omega}\right)\right)=f_{\alpha}\left(T(x)-T(x) e_{\omega}\right)$. 
Since $T(x) e_{\omega} \rightarrow_{\omega} T(x)$ and $f_{\alpha}$ is continuous, we deduce that $f_{\alpha}(T(x))=$ 0 . Now we will show that $U\left(\operatorname{ker}\left(f_{\alpha \beta}\right)\right) \subset \operatorname{ker}\left(f_{\alpha \beta}\right)$ for all $U \in M\left(E_{\beta}\right)$ and $\alpha \leq \beta$ in $\Lambda$. Since $\left(e_{\omega}\right)_{\omega \in \Omega}$ is an approximate identity in $E$ and $f_{\beta}: E \rightarrow E_{\beta}$ is a surjective continuous homomorphism, it follows that $\left(f_{\beta}\left(e_{\omega}\right)\right)_{\omega \in \Omega}$ is an approximate identity in $E_{\beta}$ (see [8, Theorem 4.1]). Let $U \in M\left(E_{\beta}\right), x_{\beta} \in$ $\operatorname{ker}\left(f_{\alpha \beta}\right)$ and $\omega \in \Omega$,

$f_{\alpha \beta}\left(U\left(x_{\beta}\right)\right)=f_{\alpha \beta}\left(U\left(x_{\beta}-x_{\beta} f_{\beta}\left(e_{\omega}\right)+x_{\beta} f_{\beta}\left(e_{\omega}\right)\right)\right)$

$=f_{\alpha \beta}\left(U\left(x_{\beta}\right)-U\left(x_{\beta} f_{\beta}\left(e_{\omega}\right)\right)\right)+f_{\alpha \beta}\left(U\left(x_{\beta} f_{\beta}\left(e_{\omega}\right)\right)\right)$

$=f_{\alpha \beta}\left(U\left(x_{\beta}\right)-U\left(x_{\beta}\right) f_{\beta}\left(e_{\omega}\right)\right)+f_{\alpha \beta}\left(x_{\beta} U\left(f_{\beta}\left(e_{\omega}\right)\right)\right)$

$=f_{\alpha \beta}\left(U\left(x_{\beta}\right)-U\left(x_{\beta}\right) f_{\beta}\left(e_{\omega}\right)\right)+f_{\alpha \beta}\left(x_{\beta}\right) f_{\alpha \beta}\left(U\left(f_{\beta}\left(e_{\omega}\right)\right)\right)$

$=f_{\alpha \beta}\left(U\left(x_{\beta}\right)-U\left(x_{\beta}\right) f_{\beta}\left(e_{\omega}\right)\right)$.

Since $U\left(x_{\beta}\right) f_{\beta}\left(e_{\omega}\right) \rightarrow_{\omega} U\left(x_{\beta}\right)$ and $f_{\alpha \beta}$ is continuous, we deduce that $f_{\alpha \beta}\left(U\left(x_{\beta}\right)\right)=0$.

Corollary 2.5. [5, Theorems 2.6 and 2.12] Let $\left(E,\left(p_{\alpha}\right)_{\alpha \in \Lambda}\right)$ be a complete locally m-pseudoconvex algebra with approximate identity. Suppose that each factor $E_{\alpha}=E / k e r\left(p_{\alpha}\right)$ in the generalized Arens-Michael decomposition of $E$ is complete. Then every multiplier of $E$ is continuous and $M(E) \cong \lim M\left(E_{\alpha}\right)$ within a topological algebra isomorphism where $M(E)$ is endowed with its pseudoseminorm topology.

Proof. It follows from Theorem 2.3 and Proposition 2.4.

Corollary 2.6. Let $\left(E,\left(p_{\alpha}\right)_{\alpha \in \Lambda}\right)$ be a locally $C^{*}$-algebra. Then every multiplier of $E$ is continuous and $M(E) \cong \lim M\left(E_{\alpha}\right)$ within a topological algebra isomorphism where $M(E)$ is endowed with its seminorm topology.

Proof. By [7, Theorem 2.6] and [10, Corollary 1.12], $E$ has an approximate identity and each factor $E_{\alpha}$ is complete.

Now we will describe multiplier algebras of complete uniform topological algebras.

Proposition 2.7. Let $\left(E,\left(p_{\alpha}\right)_{\alpha \in \Lambda}\right)$ be a uniform topological algebra. Then $\operatorname{ker}\left(f_{\alpha}\right)=\cap\left\{\operatorname{ker}(\chi): \chi \in \Delta_{\alpha}(E)\right\}$ for all $\alpha \in \Lambda$ and $\operatorname{ker}\left(f_{\alpha \beta}\right)=\cap\left\{\operatorname{ker}\left(\mu \circ f_{\alpha \beta}\right): \mu \in \Delta\left(E_{\alpha}\right)\right\}$ for all $\alpha \leq \beta$ in $\Lambda$.

Proof. Show first that $\Delta_{\alpha}(E)$ and $\Delta\left(E_{\alpha}\right)$ are non empty sets. Let $F_{\alpha}$ be the completion of $\left(E_{\alpha}, \bar{p}_{\alpha}\right), F_{\alpha}$ is a uniform Banach algebra. By [8, Lemma 5.1], $F_{\alpha}$ is commutative and semisimple. Then $\Delta\left(F_{\alpha}\right)$ is a non empty set since $F_{\alpha}$ is not a radical algebra, hence $\Delta_{\alpha}(E)$ and $\Delta\left(E_{\alpha}\right)$ are non empty sets (see [9, Proposition 7.5]).

By [1, Theorem 6], $p_{\alpha}(x)=\sup \left\{|\chi(x)|: \chi \in \Delta_{\alpha}(E)\right\}$ for all $x \in E$ and $\alpha \in \Lambda$, then $\operatorname{ker}\left(f_{\alpha}\right)=\operatorname{ker}\left(p_{\alpha}\right)=\cap\left\{\operatorname{ker}(\chi): \chi \in \Delta_{\alpha}(E)\right\}$ for all $\alpha \in \Lambda$. Let $\alpha \leq \beta$ in $\Lambda$ and $x_{\beta} \in E_{\beta}$,

$x_{\beta} \in \operatorname{ker}\left(f_{\alpha \beta}\right) \Leftrightarrow f_{\alpha \beta}\left(x_{\beta}\right)=0$ 
$\Leftrightarrow \mu\left(f_{\alpha \beta}\left(x_{\beta}\right)\right)=0$ for all $\mu \in \Delta\left(E_{\alpha}\right)$

$\Leftrightarrow x_{\beta} \in \cap\left\{\operatorname{ker}\left(\mu \circ f_{\alpha \beta}\right): \mu \in \Delta\left(E_{\alpha}\right)\right\}$.

Proposition 2.8. Let $\left(E,\left(p_{\alpha}\right)_{\alpha \in \Lambda}\right)$ be a uniform topological algebra. Then $E$ satisfies conditions (i) and $(j)$.

Proof. By Proposition 2.7, $\operatorname{ker}\left(f_{\alpha}\right)=\cap\left\{\operatorname{ker}(\chi): \chi \in \Delta_{\alpha}(E)\right\}$ for all $\alpha \in \Lambda$. If $T$ is a multiplier of $E$, then $T(k e r(\chi)) \subset \operatorname{ker}(\chi)$ for all $\chi \in \Delta_{\alpha}(E)$ by $[6$, Theorem 2.9] and [8, Lemma 5.1], so

$T\left(k e r\left(f_{\alpha}\right)\right)=T\left(\cap\left\{\operatorname{ker}(\chi): \chi \in \Delta_{\alpha}(E)\right\}\right)$

$\subset \cap\left\{T(k e r(\chi)): \chi \in \Delta_{\alpha}(E)\right\}$

$\subset \cap\left\{\operatorname{ker}(\chi): \chi \in \Delta_{\alpha}(E)\right\}=\operatorname{ker}\left(f_{\alpha}\right)$.

By Proposition 2.7, $\operatorname{ker}\left(f_{\alpha \beta}\right)=\cap\left\{\operatorname{ker}\left(\mu \circ f_{\alpha \beta}\right): \mu \in \Delta\left(E_{\alpha}\right)\right\}$ for all $\alpha \leq \beta$ in $\Lambda$. If $U$ is a multiplier of $E_{\beta}$, then $U(\operatorname{ker}(\delta)) \subset \operatorname{ker}(\delta)$ for all $\delta \in \Delta\left(E_{\beta}\right)$ by [6, Theorem 2.9] and [8, Lemma 5.1], so $U\left(k e r\left(\mu \circ f_{\alpha \beta}\right)\right) \subset \operatorname{ker}\left(\mu \circ f_{\alpha \beta}\right)$ for all $\mu \in \Delta\left(E_{\alpha}\right)$, and consequently

$U\left(\operatorname{ker}\left(f_{\alpha \beta}\right)\right)=U\left(\cap\left\{\operatorname{ker}\left(\mu \circ f_{\alpha \beta}\right): \mu \in \Delta\left(E_{\alpha}\right)\right\}\right)$

$\subset \cap\left\{U\left(\operatorname{ker}\left(\mu \circ f_{\alpha \beta}\right)\right): \mu \in \Delta\left(E_{\alpha}\right)\right\}$

$\subset \cap\left\{\operatorname{ker}\left(\mu \circ f_{\alpha \beta}\right): \mu \in \Delta\left(E_{\alpha}\right)\right\}=\operatorname{ker}\left(f_{\alpha \beta}\right)$.

Theorem 2.9. Let $\left(E,\left(p_{\alpha}\right)_{\alpha \in \Lambda}\right)$ be a complete uniform topological algebra. Then $M(E) \cong \lim M\left(E_{\alpha}\right)$ within an algebra isomorphism $\varphi$. Furthermore, if each factor $E_{\alpha}$ is complete, then every multiplier of $E$ is continuous and $\varphi$ is a topological algebra isomorphism where $M(E)$ is endowed with its seminorm topology.

Proof. It follows from Theorem 2.3 and Proposition 2.8.

Remark. Let $\left(E,\left(p_{\alpha}\right)_{\alpha \in \Lambda}\right)$ be a complete uniform topological algebra which is also a symmetric *-algebra. Then $\chi\left(x^{*}\right)=\overline{\chi(x)}$ for all $x \in E$ and $\chi \in \Delta(E)$ (see [9, Lemma 6.4]). Take $x \in E$ and $\alpha \in \Lambda$. By [1, Theorem 6], $p_{\alpha}\left(x^{*} x\right)=\sup \left\{\left|\chi\left(x^{*} x\right)\right|: \chi \in \Delta_{\alpha}(E)\right\}=\sup \left\{|\chi(x)|^{2}: \chi \in \Delta_{\alpha}(E)\right\}$ $=\left(\sup \left\{|\chi(x)|: \quad \chi \in \Delta_{\alpha}(E)\right\}\right)^{2}=p_{\alpha}(x)^{2}$.

Therefore $\left(E,\left(p_{\alpha}\right)_{\alpha \in \Lambda}\right)$ is a locally $C^{*}$-algebra, and so each factor $E_{\alpha}$ is complete.

As an application of previous results, we deduce the Arhippainen unitization theorem [1, Theorem 4] on uniform topological algebras.

Proposition 2.10. Let $\left(E,\left(p_{\alpha}\right)_{\alpha \in \Lambda}\right)$ be a uniform topological algebra, and let $M_{c}(E)$ be the algebra of all continuous multipliers of $E$. Then there is a family of seminorms $\left(q_{\alpha}\right)_{\alpha \in \Lambda}$ on $M_{c}(E)$ such that

1. $\left(M_{c}(E),\left(q_{\alpha}\right)_{\alpha \in \Lambda}\right)$ is a uniform topological algebra;

2. the map $L: E \rightarrow M_{c}(E), L(x)(y)=x y$, is an algebra isomorphism (into) and $q_{\alpha}(L(x))=p_{\alpha}(x)$ for all $x \in E$ and $\alpha \in \Lambda$. 
Proof. 1. By Propositions 2.1, 2.2 and 2.8, we define the map $\psi: M_{c}(E) \rightarrow$ $\lim _{c} M_{c}\left(E_{\alpha}\right), T \rightarrow\left(T_{\alpha}\right)_{\alpha \in \Lambda}$. As in the proof of Theorem 2.3, $\psi$ is an injective homomorphism. We endow $M_{c}(E)$ with the topology defined by the family of seminorms $q_{\alpha}(T)=\left\|T_{\alpha}\right\|_{\alpha}, \alpha \in \Lambda$, where $\|\cdot\|_{\alpha}$ is the operator norm on $M_{c}\left(E_{\alpha}\right)$. Let $T \in M_{c}(E), q_{\alpha}\left(T^{2}\right)=\left\|\left(T^{2}\right)_{\alpha}\right\|_{\alpha}=\left\|\left(T_{\alpha}\right)^{2}\right\|_{\alpha}=\left\|T_{\alpha}\right\|_{\alpha}^{2}=q_{\alpha}(T)^{2}$ since $\|\cdot\|_{\alpha}$ has the square property. Let $T \in M_{c}(E)$ with $q_{\alpha}(T)=0$ for all $\alpha \in \Lambda$, then $T_{\alpha}=0$ for all $\alpha \in \Lambda$, so $T=0$ since $\psi$ is injective.

2. Since $E$ is proper, $L$ is an algebra isomorphism (into). Let $x \in E$ and $\alpha \in \Lambda,(L(x))_{\alpha} \circ f_{\alpha}=f_{\alpha} \circ L(x)$, then $(L(x))_{\alpha}\left(f_{\alpha}(y)\right)=\left(f_{\alpha} \circ L(x)\right)(y)=$ $f_{\alpha}(x y)=f_{\alpha}(x) f_{\alpha}(y)$ for all $y \in E$. Since the $\operatorname{map} l:\left(E_{\alpha}, \bar{p}_{\alpha}\right) \rightarrow\left(M_{c}\left(E_{\alpha}\right),\|\cdot\|_{\alpha}\right)$, $l\left(x_{\alpha}\right)\left(y_{\alpha}\right)=x_{\alpha} y_{\alpha}$, is an isometric isomorphism (into), it follows that $\left\|(L(x))_{\alpha}\right\|_{\alpha}=\bar{p}_{\alpha}\left(f_{\alpha}(x)\right)=p_{\alpha}(x)$, so $q_{\alpha}(L(x))=p_{\alpha}(x)$.

Proposition 2.11. Let $E$ be a uniform topological algebra without unit, and let $E_{e}$ be the algebra obtained from $E$ by adjoining the unit. Then the map $g: E_{e} \rightarrow M_{c}(E), g((x, \lambda))=L(x)+\lambda I$ is an algebra isomorphism (into).

Proof. It is easy to show that $g$ is an algebra homomorphism. Let $(x, \lambda) \in E_{e}$ with $g((x, \lambda))=0$, then $L(x)=-\lambda I$. Suppose $\lambda \neq 0, I=-\lambda^{-1} L(x)=$ $L\left(-\lambda^{-1} x\right)$, so $-\lambda^{-1} x$ is a left unit in $E$. Since $E$ is commutative, $-\lambda^{-1} x$ is a unit in $E$, a contradiction. Thus $L(x)=0$ and consequently $x=0$ since $E$ is proper.

Corollary 2.12. [1, Theorem 4] Let $\left(E,\left(p_{\alpha}\right)_{\alpha \in \Lambda}\right)$ be a uniform topological algebra without unit. Then there is a family of seminorms $\left(s_{\alpha}\right)_{\alpha \in \Lambda}$ on $E_{e}$ such that $\left(E_{e},\left(s_{\alpha}\right)_{\alpha \in \Lambda}\right)$ is a uniform topological algebra and $s_{\alpha}((x, 0))=p_{\alpha}(x)$ for all $x \in E$ and $\alpha \in \Lambda$.

Proof. For each $\alpha \in \Lambda$, we define a seminorm on $E_{e}$ by $s_{\alpha}((x, \lambda))=q_{\alpha}(L(x)+\lambda I)$ for all $x \in E$ and $\lambda \in \mathbb{C}$.

By Propositions 2.10 and 2.11, $\left(E_{e},\left(s_{\alpha}\right)_{\alpha \in \Lambda}\right)$ is a uniform topological algebra and $s_{\alpha}((x, 0))=q_{\alpha}(L(x))=p_{\alpha}(x)$ for all $x \in E$.

Remark. We have $s_{\alpha}((x, \lambda))=q_{\alpha}(L(x)+\lambda I) \leq q_{\alpha}(L(x))+|\lambda| q_{\alpha}(I)=$ $p_{\alpha}(x)+|\lambda|$ for all $x \in E$ and $\lambda \in \mathbb{C}$. This shows that the topology on $E_{e}$ defined by the family of seminorms $\left(s_{\alpha}\right)_{\alpha \in \Lambda}$ is weaker than the usual topology on $E_{e}$ defined by the family of seminorms $\left(\tilde{p}_{\alpha}\right)_{\alpha \in \Lambda}$ where $\tilde{p}_{\alpha}((x, \lambda))=p_{\alpha}(x)+|\lambda|$.

\section{References}

[1] J. Arhippainen, On locally convex square algebras, Funct. Approx. 22 (1993), 57-63.

[2] M. Haralampidou, The Krull nature of locally $C^{*}$-algebras, Function Spaces 
(Edwardsville, IL, 2002), 195-200, Contemp. Math., 328, Amer. Math. Soc., Providence, RI, 2003.

[3] M. Haralampidou, L. Palacios, C. Signoret, Multipliers in locally convex *-algebras, Rocky Mountain J. Math., 43 (2013), 1931-1940.

[4] M. Haralampidou, L. Palacios, C. Signoret, Multipliers in perfect locally m-convex algebras, Banach J. Math. Anal., 9 (2015), 137-143.

[5] M. Haralampidou, L. Palacios, C. Signoret, Multipliers in some perfect locally $m$-pseudoconvex algebras, Proceedings of the 8 th International Conference on Topological Algebras and their Applications, 2014, Ed. by A. Katz, Series: De Gruyter Proceedings in Mathematics (to appear).

[6] T. Husain, Multipliers of topological algebras, Dissertationes Math. (Rozprawy Mat.), 285 (1989), 36 pp.

[7] A. Inoue, Locally C*-algebras, Mem. Faculty Sci. Kyushu Univ. (Ser. A), 25 (1971), 197-235.

[8] A. Mallios, Topological Algebras. Selected Topics, North-Holland, Amsterdam, 1986.

[9] E. A. Michael, Locally multiplicatively convex topological algebras, Mem. Amer. Math. Soc., 11 (1952).

[10] N. C. Phillips, Inverse limits of C*-algebras, J. Operator Theory, 19 (1988), 159-195.

Ecole Normale Supérieure

Avenue Oued Akreuch

Takaddoum, BP 5118, Rabat

Morocco

E-mail: mohammed.elazhari@yahoo.fr 